\title{
User-Query Processing through Dynamic Tweets Status Recommender System
}

\author{
V. Kakulapati ${ }^{1}$, D. Vasumathi ${ }^{2}$, G. Suryanarayana ${ }^{3}$ \\ ${ }^{1}$ SNIST, Yamnampet, Ghatkesar, Hyderabad, Telangana-501301 \\ ${ }^{2}$ JNTUCE, Kukatpally, Hyderabad, Telangana \\ ${ }^{3}$ CMRCET, kondalakoya, Medchal, Hyderabad, Telangana \\ ${ }^{1}$ vldms@yahoo.com, ${ }^{2}$ rochan44@gmail.com, ${ }^{3}$ surya.aits@gmail.com
}

\begin{abstract}
With increasing user information volume in online social networks, recommender systems have been an effective method to limit such information overload. The requirements of recommender systems specified, with widespread adoption in many internet social Twitter, Facebook, and Google online applications. In recent years, the micro-blogging in Twitter has brought greater importance to online users as a channel spreading knowledge and information. Through Twitter, users can find the relevant information on the search they perform, but understanding the past, present, and future information relevant to the investigation source is needed real-time information Estimating the successful tweet status (history, ongoing, and prospective) among the huge population of Twitter members is important to satisfy the needs of Twitter online content readers. In this paper, a Dynamic Tweets Status Recommender System (DTSRS) is designed by creating a set of dynamic recommendations to a Twitter user based on usability, consisting of people who post tweets, which is exciting present and future. The proposed recommender system is implemented through two approaches: the first is to analyze the Twitter member online tweets, select and understand the content of that tweet, and the second predicts the understanding of the tweet content, suggest the dynamic status of the tweets. In this paper, the Twitter user tweets' views are expressed after examining the depth of content, different types of user interfaces, text filtering, and machine learning technique. The set of results through tweets experimentations with database operators carried out to evaluate and comparability the proposed recommender system's performance.
\end{abstract}

Keywords

tweets, micro-blogging, context-aware, user query, recommender system

Article Received: 10 August 2020, Revised: 25 October 2020, Accepted: 18 November 2020

\section{Introduction}

With the development of internet information applications in the present technology, the social internet has become an essential behavioral channel for social users to access rapidly moving information [1]. Such social networks are the origins of many millions of social communications in the real-time world, such as blogs, reviews, tweets, updates, and much more [2][3]. The content-based recommender systems are incorporated in traditional recommender systems in an efficient approach to improve the accuracy and relevancy of the user social internet applications. Current recommender systems [4][5][6] are limited to cope up with static user preferences, could able to analyze the content adopt the learning system based on the query generated. The dynamic user preferences-based recommender system is needed to quickly and efficiently understand and find the content the user is most likely interested in and provide the recommendation based on the learning system, which is personalized.

\subsection{Motivation}

From the above issues, a Dynamic Tweets Status Recommender System provides the content based on user preference and interest by analyzing the user tweets' historical and present behavior and user tweets sharing content as relevant information. Dynamic tweets are recommended, which best suits the user query interests and tweets $\log$ information. The overload problems of data to analyze and retrieve become less and make the search engine post the user tweets based on the dynamic and personalized way, by providing explicit and exact requirements.

The Dynamic Tweets Status Recommender System is designed to quickly extract the user tweets information, with an efficient content-aware understanding, access to tweets that best-fit most from the overloaded user tweets internet information having tweets mining capability in potential user interests. The benefit of the proposed Dynamic Tweets Status Recommender System is that the user no longer needs to spend much time searching the tweets information, making the time-saving effort too low and proving the tweets' dynamics to the user, making the efforts to reduce and increases the user's satisfaction.

\subsection{Challenges}

The challenges are:

a) The tweet and retweet posts are to be analyzed, which are widespread. Proper analysis of these posts can provide the user's interactions.

b) The user interface device is length restricted to 140-characters, making the user follow the tweets independently. Specific user tweets can provide the dynamics of tweets posted.

c) The tweets' replicates are less than the unidirectional tweets, which makes the source of 
information in one direction, the importance of the tweet can be interpreted with the original tweet.

d) The user-query decision for the tweet is abundant, making the user experience ambiguity in decision- making. Filtering tweets based on personal recommendations can result in a small amount of relevant data.

\subsection{Objectives of the Proposed Work}

The contributions of the proposed Dynamic Tweets Status Recommender System are:

a) To thoroughly review the literature on the advance of deep learning and machine learning recommender systems.

b) Designing a dynamic classifier for generating tweets provides the tweets information recommendations to the interested users.

c) To design a tweets status profiler for structuring the user status through the published tweets, the user recommended followers belonging to the user's tweets content sharing interests.

d) Develop a Dynamic Tweets Status Recommender System in providing the user tweet dynamics in a

useful and privacy mode, which gives the past, present, and future tweets status.

The remaining document is structured as, Section II explains the associated work for the user-quest recommendation framework. The proposed work is listed in Section III. Section IV provides findings and debates and Section V draws conclusions.

\section{Related Works}

Recommendation Systems provide services through AI (Artificial Intelligence) and NLP (Natural Learning Processing) to provide the required evaluated explanations for the user's recommendations in several social network application networks and services [7][8]. User interface like mobile website applications enables different content related to movies, food, books, YouTube videos, health, etc. The social community moves towards movies, music, and entertainment. The recommendation system [9], based on user visibility to the content, still has security and privacy challenges. Therefore, the traditional recommendation systems [10] have become inevitable to the present user reviews and tweets in the decision making of user content [11][12].

Recommender system for Twitter with micro-blogging service makes users tweet and retweet with the length of 140 characters, named status updates of user tweets [13]. The relation between these tweets is unidirectional as the registered user tweets the tweet and another user who retweet follows the tweeting user. The list of the followed user pertains to the retweeting user only. Tweets have context and content related to the user's particular interest, such as movies, music, or specific of the said above [14]. The user who tweets is the source of information, and the user who retweet is the source of follower information, helps in analyzing the content of tweets. The micro-blogging is a way to communicate for comprehensive news spread through the user tweet channel. The Twitter users are categories into three: the first is relevant to tweets information sources, in which users are categorized into a large number of follower tweets. The second applies to tweets information seekers, in which users post a rare tweet for understanding the tweet content and follow them regularly, and the third is relevant to the relationship of user tweets [15] like friends, relatives, in which the personal tweets are retweet content.

The above categories are ranked based on the users, followers, and similar results among the tweet and retweet [16]. The ranking is made on several users, several followers, and the retweets with the recommended information sources and seekers. The orders based on these forms the popularity of relative tweets among the users and followers, including influence indicators in tweets decision making [17]. The rankings are ordered as first for tweets and retweet users, second influence user with significant influence over tweets categories, and third retweet relationship with the content of sharing in the reviews mentioned [18]. Later, these rankings are analyzed for similarity and link structure to identify the tweets network by providing proper weight to the popular tweet, which influences the connected user's ranking for different content sharing relationships [19].

The ranked tweets are focused on analyzing the URLs of the user tweets to structure the source of the tweet based on user preference and profiling with the information streamed at the recommender system based on the two interests: one is target retweet user, and the other is social content analysis with the recommended URLs for the analyzed content in the tweet recommendations [20][21]. The URLs carrying the specific tweets feeds are considered for tweet user and retweet user preferences, to analyze for mapping against their URLs tweeted and from the time of relationship made among the users in the recent tweet content ranking. The analyzed URLs address real-time tweet and retweet opinion tracking with the expressed tweets [22] with the reviews and recommendations, on the preference of information-based or content-based or with the collaborative based user tweet filtering recommendation.

In addressed URLs, the potential and relevant content of micro-blogging tweets are recommended to interested users to follow. In the recommendation procedure[23], the volume of information recommended is classified on the basis: small amount of tweet content based on the role of a user during the emergency events, medium amount of tweet content based on multiple profile strategy[24] [25] representing the broadcast of the content under the filter approach and a large amount of tweet content based on the target user in hybrid system to index the Twitter profile users through the explored range to find the accurate user through user interest assessment and personal recommendations.

Finally, the tweet content based on the profile strategy is indexed with user-queries to find tweets online on social networks. The indexed user-query recommends related tweets reflecting in different Twitter sources, with the content-based profile strategy [26] in making the user recommendations according to the observed user's interaction [27] and relationship with the structured data through dynamic user-generated content sharing from Twitter. 


\section{Proposed Work}

In this paper, a Dynamic Tweets Status Recommender System is designed through a dynamic classifier and tweets status profiler to follow the recommendation of Twitter, which provides the past, present, and future tweets status. The user's profile content includes necessary information about the user, user recommendations, user tweets, and user retweets in a dynamic classifier. The factors of user interaction time with friends, social, and relationship are analyzed. In the tweet's status profiler, user behavior and affected contextual circumstances are diagnosed with mood and social life with the geographical locations.

\subsection{Objective}

This research paper aims to provide user tweet classification based on users' social, cultural, geographical, physiological, and economic, with the user's particular needs and provide the estimation of the dynamic (i.e., past, present, and future) tweets status.

A Dynamic Tweets Status Recommender System is designed and performed sentimental analysis on user-query with tweet and retweet content processing of different tweet status changes of user query preferences and tastes to achieve this objective.

\subsection{Methodology}

To design the proposed Dynamic Tweets Status Recommender System, the methodology used are listed below:

a) To analyze the query and user profile using the representation method of annotation categories.

b) To estimate the URLs web page recommendation through popularity using the web query method of similarity degree.

c) To link the tweets and retweets relationships specific to a user social network using the followers on a social network.

d) To generate a probabilistic content model that indexes the tweets and retweets through a dynamic tweet, relevant social importance factors.

e) Training the proposed recommended system and validating the dynamic classifier using the user-tweets.

f) Compared with current recommendation structures of the calculated outcomes of the proposed systems.

Table 1 exhibits the proposed methodology

Table 1: Proposed work Methodology Steps.

\begin{tabular}{|c|c|}
\hline \multicolumn{2}{|c|}{ User-T witter-Data gathering } \\
\hline Tweets \\
\hline \multicolumn{2}{|c|}{ Pre-processing } \\
\hline Emotions \\
\hline \multicolumn{2}{|c|}{ Training data } \\
\hline Key-based tweets dataset & Cleaned tweet \\
\hline \multicolumn{2}{|c|}{ Classific ation } \\
\hline POSITIVE & NEGATIVE \\
\hline Feedback mechanism \\
\hline Sentiment scope & Relevant Process \\
\hline Recomm ender & System \\
\hline Content analysis & Behavioral analytics \\
\hline
\end{tabular}

\subsection{Design and Implementation}

The proposed Dynamic Tweets Status Recommender System uses the users shared opinions and reviews for the micro-blogging services that helps in tweets and retweets decision making under Positive, Negative and Neutrality based on extracted opinion mining, where the emotions of users are extracted by semantic analysis of the user's review by the LSTM machine learning algorithm.

The proposed Dynamic Tweets Status Recommender System is shown in figure 1 .

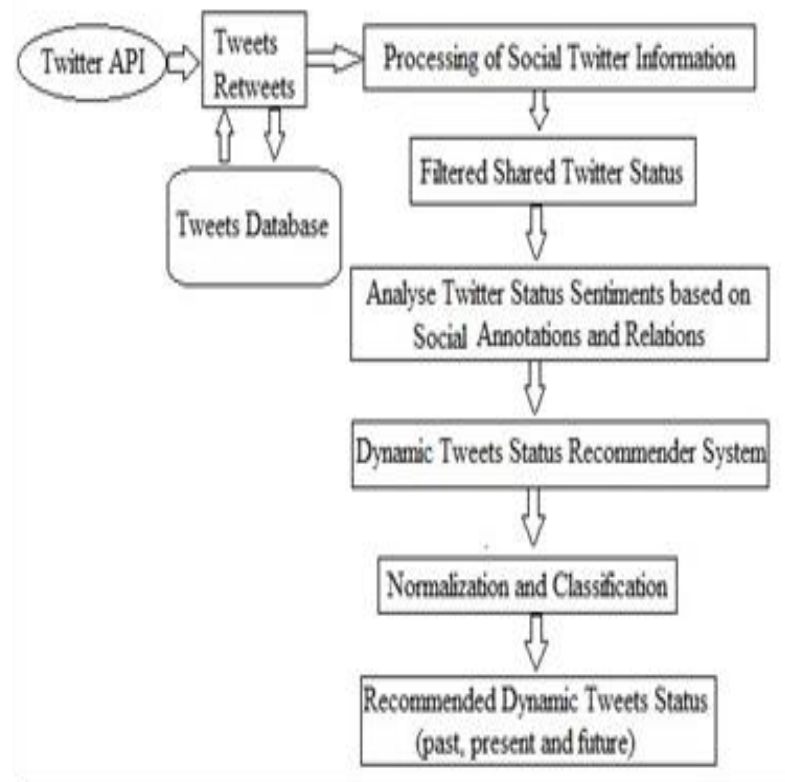

Figure 1: Proposed User Interface development diagram.

In figure 1 , the Twitter data is collected from the registered Twitter API and performs the tweets and retweets collection process through hashtags and keywords. In this work, the data were collected for the reviews of social status in 20192020. Tweets are collected based on the keywords associated with health, money, and job. The statistical tool R is used to collect the tweets and perform the analysis part required for proposed recommender system implementation. MongoDB database has been used to store the tweets, which is used as a content storage database. The tweets are retrieved from the user Twitter API and saved in MongoDB for sentiment analysis, further performing the tweets analysis.

\subsection{Dynamic Tweets Status Recommender System}




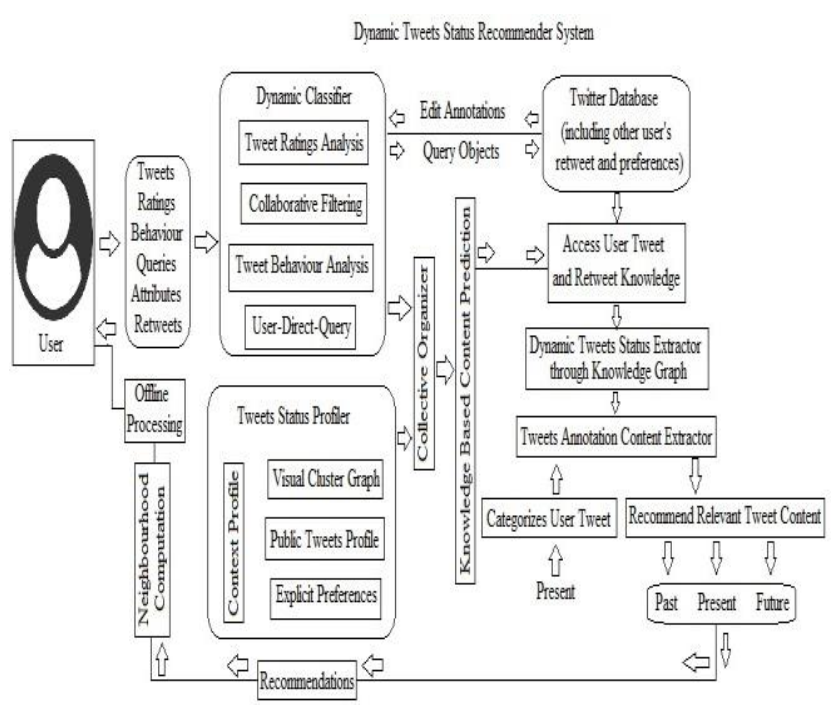

Figure 2: Proposed Dynamic Tweets Status Recommender System Flow Diagram

Figure 2 shows the proposed work; the Dynamic Classifier and Tweets Status Profiler design is shown in Algorithm 1 and 2, respectively. Algorithm 1 is responsible for limiting past tweets with recent/present tweets/user actions. This algorithm implements the userquery identity under the tweets decision tree graph. If millions of active tweets are available at this stage, they are clustered into minimal numbers based on ratings and behavior, with a minimum of up to 100 tweets. And there become the user's new tweets, forming only the present user tweets with the new list. And this method also reduces the time for extraction for similar tweets and retweets, causing to eliminate the redundancy in the tweet's submission. Algorithm 1: Dynamic Classifier

1) computeUserprofileClassQuery

2) INPUT: u (user)

3) $\mathrm{O}$ (set of past tweets)

4) OUTPUT: Ou (set of users under past tweets)

5) Begin

6) $T<-\{x$ : is a user tweet attribute $\}$

7) tuple <-pr1,...prn $\{\mathrm{pr}$ : is set of present tweets $\}$

8) $\mathrm{O}<-\{\mathrm{x}$ :tuple $\{$ set of present tweet attributes $\}\}$

9) $\quad \mathrm{A}<-\{\mathrm{x}$ :recent active past tweets $\}$

10) if $(\mathrm{A} \neq \Phi)$

11) isPresent $<=$ True

12) return isPast

13) End

Algorithm 2 is Tweets Status Profiler, the similarities in the user profiles are computed, with the given list of profiles, the similarity between the user profiles list $\mathrm{n}$ relation to the particular tweets user information is extracted and compared with the knowledge of the tweets posted earlier, to recommend the past and future tweets in the relevant knowledge graph to map with the tweet content. The algorithm is called Status Similarity, which compares with the similarity between history and future user profile tweets.

Algorithm 2: Tweets Status Profiler

1) computeStatusProfile

2) INPUT u (user)

3) $\mathrm{O}$ (set of past and present tweets)
4) OUTPUT: S (set of similar profiles)

5) Begin

6) $T<-\{x$ : is a user tweet attribute $\}$

7) $\mathrm{O}<-\{\mathrm{x}$ :tuple $\{$ set of present tweet attributes $\}\}$

8) tuple <-pa $1, \ldots$. pan $\{\mathrm{pr}$ : is set of past tweets $\}$

9) tuple $<-$ fu $1, \ldots$. fun $\{\mathrm{fu}:$ is set of future tweets $\}$

10) $\quad \mathrm{P}<$-computeProfileStatus $(\mathrm{u}, \mathrm{O})$

11) $\mathrm{A}<-\{$ set of past tweets as isActive $\}$

12) $\quad B<-\{x:$ recent future tweets $\}$

13) $\quad$ if $(B \neq \Phi)$;

14)

15)

16)

17)

18)

19)

$\forall \mathrm{s}\}$

20)

21) End

isPresent $<=$ True

return isFuture

isPresent $<=$ False

return isPast

$\mathrm{q}<=0.5$ :similarity index for user profiles

$\mathrm{S}<-\{\mathrm{s}: \mathrm{s} \&$ A AND StatusSimilarity $\{\mathrm{s}, \mathrm{u}) \geq \mathrm{q}$,

return $S$

\section{Comparative Results and Discussions}

The proposed Dynamic Tweets Status Recommender System is evaluated with online Twitter information. In the proposed work experiment, the algorithms are integrated with social networks for understanding and analyzing. The user's profiles are implemented at the user locations with the Mozilla Browser Platform. The proposed system evaluation's user profile content contains location, time, followers, relationships, age, profile summary, tweets, and retweets. The abundant user's information is passed to knowledge discovery to attain the relevant information to the recommendation system. The user interaction with the tweets and

retweets through the proposed recommendation system was provided with logins and logouts by saving them as a log file. The recommended user information is stored in its ID that offers the users recommendations with the tweets and recommended friends or recommended user retweets, concerning the time taken in the said above process called recommendation time for user tweets to analyze and predict.

\subsection{Experiments}

The proposed recommendation system is based on accuracy, which is predictive and comparative. The accuracy evaluation score states a better recommendation system. The survey literature includes several recommendation systems, having their own method of design and analysis. One standard process involves is the tweets sentiment analysis and classifier design. This is evaluated among different approaches with the proposed method, through an offline analysis, which is satisfactory to all the recommended systems. During the offline evaluation, the user tweets are attributed to the algorithms' performance, and the dataset is stored in .csv format to record the past, present, and future tweets using the conversion rate metric.

\subsection{Evaluation Metrics}

The measurement metrics for weighing, retrieving and f-measuring the proposed method accuracy. The 
accuracy, recall and f-measure relationships are given below:

Precision $=$ [TRT (total number of recommended tweets) NT (number of tweets) + TRT

Recall $=[$ TRTNNTT (number of target tweets) + TRT $] * 100$ $\%$

$\mathrm{F}-$ measure $=2 *$ [Precision $\mathrm{x}$ RecallPrecision + Recall $]$ Accuracy $=[\mathrm{TP}+\mathrm{TN} / \mathrm{TP}+\mathrm{TN}+\mathrm{FP}+\mathrm{FN}]$

where TP (True Positives), TN (True Negatives), FP (False Positives) and FN (False Negatives).

To evaluate the test results of the classifier with the sampling techniques shown in Table 2 for classifying tweets, the truth-positive, true negative, false positives, and false negative parameters are used.

Table 2: System for metric analysis Classification matrix.

\begin{tabular}{|l|l|l|}
\cline { 2 - 3 } \multicolumn{1}{c|}{} & \multicolumn{2}{|c|}{ Actual Class (Expectation) } \\
\hline Predicted Class & TP & FP \\
& Precise Result & Unexpected Result \\
\cline { 2 - 3 } & FN & TN \\
& Missing Result & $\begin{array}{l}\text { Correct absence of } \\
\text { result }\end{array}$ \\
\hline
\end{tabular}

\subsection{Analysis}

The experiments are carried through volunteer 27 users, with 17 males and 10 females Twitter user members to evaluate the proposed recommender system. The recommender system is implemented using Java SDK, WordNet Version 3.0, for classifier design and analysis. The Weka tool of version 3.6 is used, and the computer system hardware configuration tool is Intel (R) Core i5-3230M CPU @2.60 GHz with 8 GB RAM of 64-bit Operating System. The evaluation of 27 users is made by allowing them to use the proposed recommender system on their desktops to analyze their own topic or tweet information. Table 3 presents the sample of 27 users' identifications and categories.

For each user, the field of information made available in public is name, description, profile image, tweets, retweets, and profile link with the corresponding profile interests. During the implementation part, each user is made to evaluate the proposed recommendation was relevant or not and decide the system through retweets. Table 4 presents the Twitter likes of the tweets category from reviews.

Table 3: User ID and tweet category

\begin{tabular}{|c|c|c|c|}
\hline User ID & Ireet Category & Treet title & Reriens of user about Treet title \\
\hline User! & Science & The Quantum theory & Is a fimdamentat theoryinghosyics \\
\hline User? & Movile & Godzlla & Is an enomous, destuctive, prehistonc sea monster \\
\hline User12. & Spoot & Football & Modem footballonginateded Bn Bitain in the 19 th century \\
\hline Usert.9 & Envionment & Garden & Natural lemenths presentin a garden \\
\hline User2] & Space & Stars & Stars are classifed by their spectra and their temperature \\
\hline
\end{tabular}

Table 4: User ID, tweet category from twitter likes.

\begin{tabular}{|l|l|l|l|}
\hline User ID & Tweet Category & Semantic Emotions & Twitter likes \\
\hline User1 & Science & Highly Favourable & $31567 \mathrm{~K}$ \\
\hline User7 & Movie & Highly Favourable & $56489 \mathrm{~K}$ \\
\hline User12 & Sport & Average Favourable & $21359 \mathrm{~K}$ \\
\hline User19 & Environment & Highly Favourable & $49125 \mathrm{~K}$ \\
\hline User27 & Space & Average Favourable & $20458 \mathrm{~K}$ \\
\hline
\end{tabular}

Table 5 and Table 6 presents the comparison of the proposed recommender system and other survey models mobile movie recommendation service [MMRS] [15], sentiment-enhanced hybrid recommender system [SHRS][24], movie recommendation system [MRS][19], a deep temporal neural music recommendation model [DNMRM][8], multivariant expert system [MES][14]and multivariate System [MVS][16]. The F score results for the proposed recommender system are compared with other parameters and other recommendation systems. The accuracy of the proposed Dynamic Tweets Status Recommender System is $99.1 \%$, stating the tweets categories are genuinely interested in users. With a true positive rate of $99.46 \%$, the recommendation system makes interest to those intended and recommended users only.

Table 5: A comparison of the various judgment criteria of the recommendation.

\begin{tabular}{|l|c|c|c|c|}
\hline Decision Parameters & TP & TN & FP & FN \\
\hline MES & 442 & 387 & 114 & 57 \\
\hline SHRS & 375 & 355 & 95 & 175 \\
\hline MRS & 406 & 347 & 116 & 131 \\
\hline DNMRM & 525 & 250 & 90 & 135 \\
\hline MMRS & 341 & 237 & 207 & 215 \\
\hline MVS & 554 & 433 & 8 & 5 \\
\hline Proposed DTSRS & 556 & 435 & 6 & 3 \\
\hline
\end{tabular}

Table 6: Results of the experiments.

\begin{tabular}{|l|l|l|l|l|l|l|l|}
\hline & IES & SERS & IIRS & DNMIRI & IIIRS & MIS & Proposed DTSRS \\
\hline Precision & 0.7950 & 0.7979 & 0.7778 & 0.8537 & 0.6223 & 0.9858 & 0.9893 \\
\hline Recall & 0.8858 & 0.6818 & 0.7561 & 0.7955 & 0.6133 & 0.9911 & 0.9946 \\
\hline F score & 0.8379 & 0.7353 & 0.7668 & 0.8235 & 0.6178 & 0.9884 & 0.9919 \\
\hline Accuracy & 0.8290 & 0.7300 & 0.7530 & 0.7750 & 0.5780 & 0.9870 & 0.9910 \\
\hline
\end{tabular}

\section{Conclusions}

In this paper, a Dynamic Classifier and Tweets Status Profiler algorithms are proposed to design Dynamic Tweets Status Recommender System. The experiments are implemented with the user tweets in a Java environment with a classifier library to improve the response time and generate the proper recommendations. During the implementation, the proposed recommender system used opinion polling and sentiment analysis to extract user past, present, and future recommender tweets in order and rank based. Therefore, significant recommendations are derived efficiently using the LSTM learning algorithm, which increased tweets' classification towards the past, present, and future user tweets categories mentioned in the simulation analysis. The simulation results of experiments show the proposed recommender system has comparative improvements of the 
recommender systems to apply in the tweets sentence-level and content level.

\section{Future Work}

The proposed Dynamic Tweets Status Recommender System can be further enhanced by including more user tweet profile parameters like movie playlist, Twitter user groups, tweets sessions, user emotions, user tweet tags, and the feature retweet content to improve the recommender system..

\section{References}

[1] Chen, J., Nairn, R., Nelson, L., Bernstein, M., Chi, E.: Short and tweet: experiments on recommending content from information streams. In: Proc. of the 28th Int. Conf. on Human Factors in Computing Systems (CHI'10). pp. 1185-11942010.

[2] Crisci, V. Grasso, P. Nesi et al., "Predicting TV programme audience by using Twitter based metrics," Multimedia Tools and Applications, vol. 77, no. 3, pp. 12203-12232, 2018.

[3] E. Mohammadi, M. elwall, M. Kwasny, and K. L. Holmes, "Academic information on twitter: a user survey," PLoS One, vol. 13, no. 5, Article ID e0197265, 2018.

[4] Esparza, S.G., O’Mahony, M.P., Smyth, B.: On the real-time web as a source of recommendation knowledge. In: Proc. of the 4th ACM Conf. on Recommender Systems (RecSys'10). pp. 305-308 2010.

[5] F. Emmert-Streib, O. P. Yli-Harja, M. Dehmer, and F. Emmert-Streib, "Data analytics applications for streaming data from social media: what to predict?" Frontiers in Big Data, vol. 1, p. 2, 2018.

[6] Garcia, R., Amatriain, X.: Weighted content based methods for recommending connections in online social networks. In: Workshop on Recommender Systems and the Social Web. pp. 68-71. Barcelona, Spain 2010.

[7] H. Li, D. Caragea, C. Caragea, and N. Herndon, "Disaster response aided by tweet classification with a domain adaptation approach," Journal of
Contingencies and Crisis Management, vol. 26, no. 1, pp. 16-27, 2018.

[8] H.-T. Zheng, J.-Y. Chen, N. Liang, A. K. Sangaiah, Y. Jiang, and C.-Z. Zhao, "A deep temporal neural music recommendation model utilizing music and user metadata," Applied Science, vol. 9, no. 4, p. 703, 2019.

[9] Java, A., Song, X., Finin, T., Tseng, B.: Why we twitter: understanding microblogging usage and communities. In: Proc. of the 9th WebKDD and 1st SNAKDD 2007 Workshop on Web Mining and Social Network Analysis. pp. 56-65 2007.

[10] Joachims, T., Granka, L., Pan, B., Hembrooke, H., Gay, G.: Accurately interpreting click through data as implicit feedback. In: Proc. of the 28th Int. ACM SIGIR Conf. on Research and Development in Information Retrieval (SIGIR'05). pp. 154-161 2005.

[11] Kwak, H., Lee, C., Park, H., Moon, S.: What is Twitter, a social network or a news media? In: Proc. of the 19th Int. Conf. on World Wide Web (WWW'10). pp. 591-600 2010.

[12] Liben-Nowell, D., Kleinberg, J.: The link prediction problem for social networks. In: Proc. of the 12th Int. Conf. on Information and knowledge management. pp. 556-559. CIKM '03, ACM, New York, NY, USA 2003.

[13] M. Gao and X. Zhang, A movie recommender system from tweets data, January 2019, http://cs229.stanford.edu/proj2015/ 299_report.pdf.

[14] M. Ibrahim and I. Bajwa, "Design and application of a multivariant expert system using apache hadoop framework," Sustainability, vol. 10 , no. 11 , p. 4280 , 2018.

[15] M.-Y. Hsieh, W.-K. Chou, and K.-C. Li, "Building a mobile movie recommendation service by user rating and APP usage with linked data on Hadoop," Multimedia Tools and 
Applications, vol. 76, no. 3, pp. 33833401, 2017.

[16] Muhammad Ibrahim, Imran Sarwar Bajwa , Riaz Ul-Amin, and Bakhtiar Kasi, " A Neural Network-Inspired Approach for Improved and True Movie Recommendations", Hindawi, Computational Intelligence and Neuroscience, Volume 2019, pp-1-19.

[17] O. Loyola-Gonz'alez, A. L'opez-Cuevas, M. A. Medina-P'erez et al., "Fusing pattern discovery and visual analytics approaches in tweet propagation," Information Fusion, vol. 46, pp. 91101,2018 .

[18] Phelan, O., McCarthy, K., Smyth, B.: Using Twitter to recommend real-time topical news. In: Proc. of the 3rd ACM Conf. on Recommender Systems (RecSys'09). pp. 385-388 2009.

[19] S. Kumar, S. S. Halder, K. De, and P. P. Roy, "Movie recommendation system using sentiment analysis from microblogging data," 2018, https://arxiv.org/abs/1811.10804.

[20] S. Stieglitz, M. Mirbabaie, B. Ross, and C. Neuberger, "Social media analytics - challenges in topic discovery, data collection, and data preparation," International Journal of Information Management, vol. 39, pp. 156-168, 2018.

[21] Tang, J.; Belletti, F.; Jain, S.; Chen, M.; Beutel, A.; Xu, C.; Chi, E.H. Towards Neural Mixture Recommender for Long Range Dependent User Sequences. In Proceedings of the World Wide Web Conference, WWW 2019, San Francisco, CA, USA, 13-17 May 2019; pp. 17821793.

[22] Wang, S.; Cao, L.;Wang, Y. A Survey on Session-based Recommender Systems. arXiv 2019, arXiv:1902.04864.

[23] Wu, S.; Tang, Y.; Zhu, Y.; Wang, L.; Xie, X.; Tan, T. Session-Based Recommendation with Graph Neural Networks. In Proceedings of The Thirty-Third AAAI Conference on Artificial Intelligence, AAAI 2019,
Honolulu, HI, USA, 27 January-1 February 2019; pp. 346-353.

[24] Y. Wang, M. Wang, and W. Xu, "A sentiment-enhanced hybrid recommender system for movie recommendation: a big data analytics framework," Wireless Communications and Mobile Computing, vol. 2018, Article ID 8263704, 9 pages, 2018.

[25] Y.Wu, M. Qi, and R. Yang, “A news recommendation system based on an improved collaborative ltering algorithm," (in Chinese), Comput. Eng. Sci., vol. 39, no. 6, pp. 11791185, 2017.

[26] Yuan, F.; Karatzoglou, A.; Arapakis, I.; Jose, J.M.; He, X. A Simple Convolutional Generative Network for Next Item Recommendation. In Proceedings of the Twelfth ACM International Conference on Web Search and Data Mining, WSDM 2019, Melbourne, Australia, 11-15 February 2019; pp. 582-590.

[27] Zhang, S.; Yao, L.; Sun, A.; Tay, Y. Deep Learning Based Recommender System: A Survey and New Perspectives. ACM Comput. Surv. 2019, 52, 5:1-5:38. 